\title{
Editorial of Virtual Special Issue Frontiers in Water Biophysics 2017
}

The present virtual special issue (VSI) of the Journal of Molecular Liquids contains the proceedings of the 4th Conference on Frontiers in Water Biophysics (FWB2017) held in Erice, Sicily (Italy) from 23 to 27 May 2017 at the Ettore Majorana Foundation and Centre for Scientific Culture, in the frame of the V Course of the International School of Sta- tistical Physics (Directors: P. Hanggi, F. Marchesoni).

As for the past editions, the FWB2017 conference was aimed at offering opportunities for presentation and discussion of the recent research in the field of water structure and dynamics at the nano- scale and mesoscale, in relation with the active role played by water in food, pharmaceutical and life sciences, and on their re- lated technological aspects. In 2017 special emphasis was given to hydration properties of biological systems, with particular focus on the case of proteins.

More than 60 scientific participants, including $\mathrm{PhD}$ students and postdoctoral researchers, from 14 countries attended the conference with a total of 50 presentations, encompassing tutorials, plenary and in- vited lectures, oral and poster presentations. The organizers acknowl- edge the continuous advice of the Ettore Majorana Center and they are grateful to all the sponsors of the conference: Elettra-Sincrotrone - Trieste; Department of Physics and Chemistry - University of Palermo; Italian CNR-IOM; Institute of Nanoscience and Nanotechnology (IN2UB) - Universitat de Barcelona; University of Trieste; Department of Physics and Geology - University of Perugia.

This virtual special issue encompasses thirteen selected contribu- tions in which participants of the FWB2017 employed experimental, theoretical or computational methods, providing original results, out- looks, methods and/or interpretations helpful to improve our knowl- edge on fundamental aspects of water properties and on their connection with bio-related systems of different nature and complexity.

In particular, Pica and Graziano showed how the pressure depen- dence of the cavity contact correlation function of water, an important structural quantity implicated in the hydration thermodynamics of non- polar solutes, can be reasonably well reproduced in the framework of the classic scaled particle theory (SPT) based on the experimental den- sity of water [1].

Employing a combination of molecular mechanics and ab initio cal- culations, Hassanali and coworkers investigated hydrogen bonding in peptide-peptide and peptide-water clusters relevant in protein aggre- gation. In particular, the role of quantum mechanical effects and that of three-body interactions has been explored [2].

Grimaldi and Graziano discussed and reviewed a theoretical ap- proach, based to solventexcluded volume effects, which has proven the capability of explaining the role of water on both hot and cold dena- turation of small globular proteins [3]. 
Within the ambit of protein purification technology, Coluzza and co- workers studied the translocation of a globular polymer through a cylin- drical pore internally coated with a polymer brush by extended numerical simulations [4].

An outlook on the relevance of the nonergodic exploration of the protein configuration space on determining the activation barrier in protein electron transfer is given by Matyushov. The role played by the protein-water interface in this context has been also discussed [5].

Corezzi and coworkers determined the acoustic properties of an aqueous suspension of polyN-isopropylacrylamide (PNIPAM) microgels employing a new strategy to analyze Brillouin spectra of opaque samples [6].

The protein dynamical transition (PDT) and its correlation with the external medium have been investigated by Librizzi and coworkers using temperature dependent elastic neutron scattering (ENS) and IR absorption. Experimental data on myoglobin in water-glycerol, indi- cates the occurrence of coupling between the dynamics of protein back- bone and the fluctuations of amino acid side chains, both processes are thought to be mediated by the solvent [7].

The influence of the model protein lysozyme on structural and dy-namical properties of surrounding water has been investigated by Gallo and coworkers by means of molecular dynamics (MD) simula- tions. Among other results, this study reveals an extreme slowing down of water relaxation in the first protein hydration layer [8].

UV Raman, Brillouin light scattering (BLS) and Small Angle Neutron Scattering (SANS) have been employed by Rossi and coworkers to gain insights on structural and viscoelastic properties of polymeric cyclodextrin-based nanosponges hydrogels in response to $\mathrm{pH}$ varia- tions. Concomitant changes on the organization of water embedded within the nanosponge matrix are also evidenced [9].

Tadini and coworkers investigated the osmotic dehydration of slices of cambuci fruits with binary (sorbitol-water) and ternary (sorbitol calcium lactate) solutions, relevant in food dehydration pro- cesses. The equilibrium moisture and soluble solids content were esti- mated, and a reduction of the effective diffusion coefficient of water upon addition of calcium lactate was observed [10].

Biomolecular surfaces in air absorb small amount of water whose relevant wetting properties at nanoscales have not been investigated so far. Bittner and coworkers have characterized wetting features of a model virus by ionic liquids at very high spatial resolution by using AFM, SEM and STEM (scanning transmission electron microscopy). The authors discussed to what extent their findings can be extended to liquid water [11].

To shed lights into how the regions of correlated hydrogen bonds could lead to the occurrence of a liquid-liquid critical point (LLCP) in the deep supercooled region of water, Bianco 
and Franzese considered a theoretical model for a monolayer of liquid water, mapping cluster formation and percolation features in a broad range of temperatures and pressures via Monte Carlo simulations. Results are discussed in relation with the relevant thermodynamics [12].

Finally, Capaccioli and coworkers analyzed quasi-elastic neutron scattering (QENS), Mössbauer, and Brillouin light scattering data of a range of dry proteins, and demonstrated the existence of a novel dy- namical transition at around $200 \mathrm{~K}$. The authors argued that this transi- tion is independent on the time window probed by the experiment, differing from the well-known dynamical transition observed in hy- drated proteins. The novel transition does not depend on the trace amount of water in the sample and its presence might have implications on the long-term stability of lyophilized proteins [13].

We want to thank all the authors who have contributed to this vir- tual special issue and the reviewers for their effort in providing accurate evaluations of the papers and their improvement. A special acknowl- edgement is devoted to Prof. Wolffram Schröer (JML Editor in Chief) for his priceless support and to Emma Xu (Publishing Content Specialist, Elsevier) for her precious help during the preparation of this VSI.

Marco Paolantoni Dipartimento di Chimica, Biologia e Biotecnologie (DCBB), Università degli Studi di Perugia, Italy E-mail address: marco.paolantoni@unipg.it

Attilio Cesàro Elettra-Sincrotrone Trieste, Italy

Lucia Comez CNR-IOM, clo Dipartimento di Fisica e Geologia, Università degli Studi di Perugia, Italy

Giancarlo Franzese Departament de Física de la Matèria Condensada \& Institute of Nanoscience and Nanotechnology (IN2UB), Universitat de Barcelona, Spain 


\section{References}

[1] A. Pica, G. Graziano, On the ability of classic scaled particle theory to reproduce the cavity contact correlation function of water over a large hydrostatic pressure range, J. Mol. Liq. 263 (2018) 243-246.

[2] K. Jong, N. Ansaria, L. Grisanti, A. Hassanali, Understanding the quantum mechanical properties of hydrogen bonds in solvated biomolecules from cluster calculations, J. Mol. Liq. 263 (2018) 501-509.

[3] A. Grimaldi, G. Graziano, Water and cold denaturation of small globular proteins, J. Mol. Liq. 264 (2018) 579-584.

[4] A. Mair, C. Tung, A. Cacciuto, I. Coluzza, Translocation of a globular polymer through a hairy pore, J. Mol. Liq. 265 (2018) 603-610.

[5] D.V. Matyushov, Fluctuation relations, effective temperature, and ageing of enzymes: The case of protein electron transfer, J. Mol. Liq. 266 (2018) 361-372.

[6] S. Corezzi, L. Comez, M. Zanatta, A simple analysis of Brillouin spectra from opaque liquids and its application to aqueous suspensions of poly- $\mathrm{N}$-isopropylacrylamide microgel particles, J. Mol. Liq. 266 (2018) 460-466.

[7] F. Librizzi, A. Caliò, A. Cupane, Dynamical properties of myoglobin in an ultraviscous waterglycerol solvent investigated with elastic neutron scattering and FTIR spectroscopy, J. Mol. Liq. 268 (2018) 242-248.

[8] G. Camisasca, A. Iorio, M. De Marzio, P. Gallo, Structure and slow dynamics of protein hydration water, J. Mol. Liq. 268 (2018) 903-910.

[9] B. Rossi, C. Bottari, L. Comez, S. Corezzi, M. Paolantoni, A. Gessini, C. Masciovecchio, A.Mele, C. Punta, L. Melone, A. Fiorati, A. Radulescu, G. Mangiapia, A. Paciaroni, Structural and molecular response in cyclodextrin-based $\mathrm{pH}$-sensitive hydrogels by the joint use of Brillouin, UV Raman and Small Angle Neutron Scattering techniques, J. Mol. Liq. 271 (2018) 738-746.

[10] M.S. Paes, J.P.F. Del Pintor, P.D. Pessoa, C.C. Tadini, Mass transfer modeling during osmotic dehydration of cambuci (Campomanesia phaea (O. Berg) Landrum) slices and quality assessment, J. Mol. Liq. 273 (2018) 408-413.

[11] J.M. Alonso, T. Ondarçuhu, C. Parrens, M. Górzny, A.M. Bittner, Nanoscale wetting of viruses by ionic liquids, J. Mol. Liq. 276 (2018) 667-674.

[12] V. Bianco, G. Franzese, Hydrogen bond correlated percolation in a supercooled water monolayer as a hallmark of the critical region, J. Mol. Liq. 285 (2019) 727-739.

[13] K.L. Ngai, Liang Hong, S. Capaccioli, A. Paciaroni, Uncovering a novel transition in the dynamics of proteins in the dry state, J. Mol. Liq. In press 